\section{Estructura y funciones de la Atención Primaria de Salud según el Primary Care Assessment Tool para prestadores en la comuna de Conchalí - Santiago de Chile}

\author{
KAREN PESSE-SORENSEN ${ }^{1, \mathrm{c}}$, ALEJANDRA FUENTES-GARCÍA $^{2, \mathrm{a}, \mathrm{d}}$, \\ JUAN ILABACA ${ }^{2, b}$
}

\section{Primary Care Assessment Tool applied to primary health care workers from Conchalí, Santiago}

Background: The Chilean public health system is based on Primary Health Care (PHC), whose assessment is challenging due to the heterogeneity of services and multi-dimensionality of expected results. The Primary Care Assessment Tool was adapted and validated for Chile. Aim: To analyze, from the provider's perspective, the structure and functioning of the health centers, to determine the achievement of PHC's core functions: access, continuity, coordination, comprehensiveness of care, cultural competence, family centeredness and community orientation. Material and Methods: All professionals working in primary care in a commune of Metropolitan Santiago were invited to answer an online version of the Primary Care Assessment Tool. Results: One hundred and nine professionals (51\% of those invited) from four Family Health Centers, two Community Health Centers, and a Community Mental Health Center, answered the online questionnaire. Their distribution by profession and health units does not resemble the whole research population, which should be considered when interpreting the results. Data show a good performance of the system: general and domain specific scores are all near three for a maximum score of four. Family centeredness obtained the highest score, whereas cultural competence had the lowest. Conclusions: Reinforcing intercultural skills and a wider approach to psycho-social problems is recommended to strengthen the new healthcare model implementation.

(Rev Med Chile 2019; 147: 305-313)

Key words: Community Health Centers; Health Personnel; Primary Health Care; Quality of Health Care.

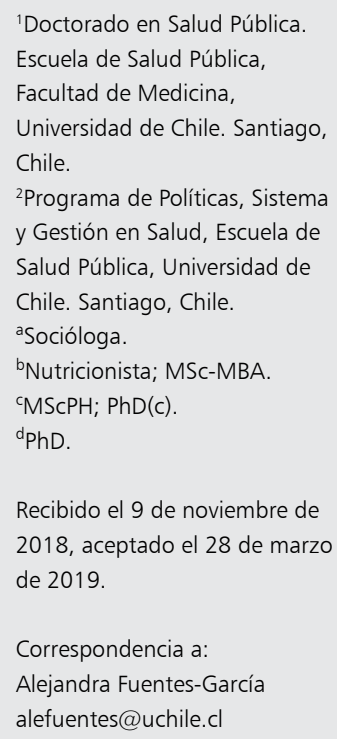

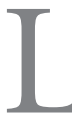

a Atención Primaria de Salud (APS) constituye la base del sistema público de salud chileno. El Modelo de Salud Integral, Familiar y Comunitaria (MAIS) responde a las necesidades de sus usuarios, para lo cual la atención debe ser integral, oportuna, de calidad y resolutiva, y culturalmente aceptada por la población, fomentando su participación e incluyendo otros sectores ${ }^{1}$. La familia es considerada como unidad primaria en que se promueve la salud y se tratan enfermedades $^{2}$. La integralidad, referida a los niveles de prevención y al abordaje multidimensional de los problemas, la continuidad del cuidado y la coordinación intra- e interinstitucional, son ele- 
mentos estructurantes del modelo ${ }^{1}$. Estos valores, principios y elementos también son propuestos en la versión renovada de APS de la Organización Panamericana de la Salud ${ }^{3}$.

Dada la heterogeneidad y complejidad de los servicios ofrecidos y la multidimensionalidad de los resultados esperados, la evaluación de la APS es compleja. Numerosas publicaciones destacan la importancia de incluir las percepciones de los involucrados en la evaluación de la calidad ${ }^{4-6}$, aunque la tendencia ha sido descuidar la mirada holística sobre el funcionamiento del sistema y el involucramiento de sus principales actores y de otros sectores ${ }^{7}$.

El Centro de Políticas de Atención Primaria de la Universidad Johns Hopkins diseñó un conjunto de instrumentos que permiten caracterizar el funcionamiento de la APS $^{8}$. Los cuestionarios PCAT (Primary Care Assessment Tool) exploran cuatro dominios: acceso y primer contacto, continuidad, coordinación e integralidad; y tres características claves: competencia cultural, enfoque familiar y orientación comunitaria y están dirigidos a usuarios infantiles y adultos, prestadores y gestores ${ }^{9}$.

Los cuestionarios PCAT fueron adaptados para diversos países y contextos socioculturales ${ }^{8,10-12}$. Brasil fue el primer país latinoamericano en evaluar su consistencia interna y confiablidad ${ }^{13}$, seguido por Uruguay ${ }^{14}$ y Argentina ${ }^{15}$. En Chile, los instrumentos para usuarios adultos y prestadores fueron adaptados en 2016; el PCAT-Prestadores fue validado en diferentes regiones, incorporando a todos los profesionales del equipo de salud ${ }^{16}$.

Aunque el MAIS cuenta con sus propios instrumentos e indicadores de evaluación ${ }^{17-20}$, y se han propuesto otras dimensiones e instrumentos para medir la calidad de la atención ${ }^{21,22}$, el PCAT detalla el funcionamiento de la APS desde la percepción de los involucrados.

Este artículo describe la aplicación del PCAT-Prestadores en la comuna de Conchalí. Desde esta perspectiva, se analiza la estructura y funcionamiento de sus establecimientos de APS respecto del cumplimiento de sus funciones esenciales.

\section{Material y Métodos}

Estudio descriptivo cuantitativo realizado en los centros APS de Conchalí, Región Metropolitana. En esta comuna, $81,7 \%$ de su población está adscrita al Fondo Nacional de Salud ${ }^{23}$ y 21,6\% está en situación de pobreza multidimensional ${ }^{24}$ con un perfil similar al nacional ${ }^{25}, 14,5 \%$ de los habitantes de la comuna tiene 65 o más años, y más de la mitad $(58,2 \%)$ de ellos presenta sobrepeso u obesidad. La primera causa de muerte la constituyen enfermedades del sistema circulatorio ${ }^{26}$.

Dos centros comunitarios de Salud Familiar (CECOSF); cuatro centros de Salud Familiar (CESFAM), un centro comunitario de Salud Mental (COSAM), un centro comunitario de Rehabilitación, dos servicios de Atención Primaria de Urgencia y un servicio de urgencia de alta resolutividad ${ }^{27}$ atienden a esta población. Trabajan 214 profesionales y 114 técnicos, además de administrativos.

\section{Universo y muestra}

En octubre de 2017 se invitó a todos los profesionales que trabajaban en los CESFAM, CECOSF y COSAM a participar en el estudio. Se envió un mensaje inicial y dos recordatorios posteriores a sus correos electrónicos. Se solicitó a los directores de establecimientos que informaran a sus equipos sobre la investigación, sugiriendo que liberaran tiempo para responder el cuestionario; lo que fue reforzado por la investigadora principal durante una jornada de capacitación continua al personal, logrando un incremento de $10 \%$ en la tasa de respuesta.

Se excluyó al personal que trabaja en los servicios de urgencia y de rehabilitación, ya que los criterios de esta evaluación no resultaban pertinentes para ellos. El COSAM fue incluido aun siendo un establecimiento especializado en salud mental, ya que su funcionamiento se enmarca en casi todas las dimensiones del PCAT.

\section{Recolección y análisis de la información}

La información fue recogida entre octubre de 2017 y mayo de 2018 mediante una versión digital del instrumento PCAT-Prestadores, previamente adaptado y validado para la realidad chilena ${ }^{16}$, enviado por GoogleForms. La revisión de consistencia interna y el análisis de datos fue realizado con Stata versión $13.1^{\circledR}$.

Siguiendo la guía de uso del PCAT ${ }^{16}$, a cada respuesta se le asignó un puntaje en una escala de 1 ("definitivamente no") a 4 puntos ("definitivamente sî"). A las respuestas "no estoy seguro/ 
no sé" se les asignó el valor 2 (correspondiente a "probablemente no") cuando representaban menos de 50\% de los ítems del subdominio. A partir de los puntajes de cada una de las dimensiones y subdimensiones incluidas en el cuestionario, se obtuvo el puntaje esencial, que es el promedio de los puntajes obtenidos en las cuatro dimensiones principales (primer contacto, continuidad, coordinación, integralidad), y el puntaje global, que incluye todas las dimensiones. No existe una escala consensuada internacionalmente para valorar los puntajes obtenidos en el PCAT. La guía de uso recomienda considerar como buen desempeño los puntajes iguales o mayores a tres puntos, que corresponden a la respuesta "probablemente sí", indicando el cumplimiento de la función evaluada ${ }^{16}$.

Los resultados preliminares fueron presentados a los actores locales, quienes manifestaron la coincidencia de estos con su percepción del funcionamiento de la APS en la comuna.

\section{Consideraciones éticas}

Esta investigación fue aprobada por el Comité de Ética de Investigación en Seres Humanos de la Facultad de Medicina de la Universidad de Chile, por la autoridad del Servicio de Salud Metropolitano Norte y el CORESAM Conchalí. El cuestionario incluía un consentimiento informado con la aceptación de participar voluntariamente.

\section{Resultados}

Se obtuvieron 109 respuestas, lo que representa $50,94 \%$ de los profesionales incluidos en el estudio. La Tabla 1 muestra la distribución de estos según profesión y tipo de establecimiento. Los nutricionistas y psicólogos tuvieron mayores porcentajes de respuesta; los kinesiólogos y médicos los menores. Los CESFAM tienen bajas tasas de respuesta.

La mayoría $(55,1 \%)$ de los profesionales que contestaron la encuesta trabajaba en APS desde hacía más de cinco años, 77,1\% lo hacía en jornada completa y $85,3 \%$ recibía su remuneración como sueldo. El 90,8\% reportó ser elegible para los estímulos al desempeño por buen trato y por cumplimiento de metas sanitarias.

La Tabla 2 resume la percepción de los prestadores sobre las características de la población atendida.

El puntaje global obtenido por el sistema APS de Conchalí en el PCAT fue de 3,12 puntos ( IC $_{95 \%}$ : 3,06-3,17), el puntaje esencial fue $3,08\left(\mathrm{IC}_{95 \%}\right.$ : $^{2}$ : 3,02-3,13). La valoración más alta corresponde al dominio enfoque familiar que obtuvo un promedio de 3,48 ( $\mathrm{IC}_{95 \%}$ 3,39-3,57); cercano al de coordinación en sistema de información $(3,36$; $\mathrm{IC}_{95 \%:}$ 3,26-3,45). El aspecto con menor puntaje fue competencia cultural $\left(2,79 ; \mathrm{IC}_{95 \%}\right.$ : 2,69-2,89).

En la Tabla 3 se aprecia que al separar los datos

Tabla 1. Profesión y tipo de establecimiento en que trabajan los encuestados

\begin{tabular}{|c|c|c|c|c|c|c|c|c|c|c|c|c|}
\hline \multirow[b]{2}{*}{ Centro } & \multicolumn{12}{|c|}{ Profesión } \\
\hline & 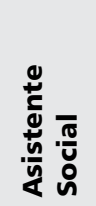 & 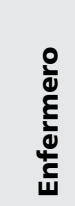 & $\begin{array}{l}\circ \\
\text { 음 } \\
\frac{0}{0} \\
\frac{y}{y} \\
\frac{5}{x}\end{array}$ & $\frac{c}{0}$ & 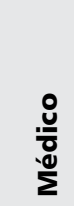 & 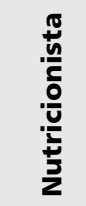 & $\begin{array}{l}\text { 음 } \\
\text { 응 } \\
\text { 홍 } \\
\text { 응 }\end{array}$ & $\begin{array}{l}\circ \\
\circ \\
\frac{0}{0} \\
\frac{u}{n}\end{array}$ & $\begin{array}{l}\text { * } \\
\text { ถ้ } \\
\text { o }\end{array}$ & $\begin{array}{l}\bar{\varpi} \\
\stackrel{0}{0}\end{array}$ & 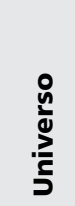 & 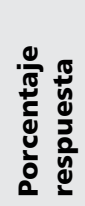 \\
\hline CECOSF & 3 & 3 & 1 & 1 & 2 & 1 & 1 & 1 & - & 13 & 22 & 59,1 \\
\hline CESFAM & 5 & 13 & 7 & 13 & 12 & 6 & 11 & 9 & - & 76 & 164 & 46,3 \\
\hline COSAM & 4 & - & - & - & - & - & - & 11 & 5 & 20 & 26 & 76,9 \\
\hline Total & 12 & 16 & 8 & 14 & 14 & 7 & 12 & 21 & 5 & 109 & & \\
\hline Universo & 19 & 34 & 25 & 24 & 40 & 11 & 24 & 29 & $8 * *$ & & 214 & \\
\hline Porcentaje respuesta & 63,2 & 47,1 & 32,0 & 58,3 & 35,0 & 63,6 & 50,0 & 72,4 & 71,4 & 50,9 & & \\
\hline
\end{tabular}

*Fuente: Registro CORESAM. **6 Terapeutas ocupacionales y dos educadoras de párvulo de Chile Crece Contigo. Fuente: PCAT Prestadores, Conchalí 2017-18. 
Tabla 2. Características de la población atendida según los prestadores

\begin{tabular}{|c|c|c|}
\hline Característica & Indicador & $\begin{array}{l}\text { Respuestas } \\
\text { encuestados }\end{array}$ \\
\hline Pacientes con problemas de larga duración o discapacidad & $\geq 61 \%$ & $37,6 \%$ \\
\hline $\begin{array}{l}\text { Continuidad intra-prestador: Tiempo que pacientes permanecen siendo atendidos } \\
\text { por prestador }\end{array}$ & $\begin{array}{l}\leq 1 \text { año } \\
\text { Muy variable }\end{array}$ & $\begin{array}{l}38,5 \% \\
31,2 \%\end{array}$ \\
\hline A cargo de una población geográficamente definida & Sí & $87,2 \%$ \\
\hline $\begin{array}{l}\text { Pacientes en lista o registro computarizado que los identifica como pacientes del } \\
\text { prestador }\end{array}$ & $\geq 61 \%$ & $83,5 \%$ \\
\hline $\begin{array}{l}\text { Integralidad: Pacientes que usan la consulta del prestador para todas sus necesidades } \\
\text { de atención }\end{array}$ & $\geq 61 \%$ & $53,2 \%$ \\
\hline
\end{tabular}

Fuente: PCAT Prestadores, Conchalí 2017-18.

Tabla 3. Puntajes promedio globales y por dominio

\begin{tabular}{|lcccc|}
\hline & \multicolumn{3}{c|}{ Tipo establecimiento } \\
Dimensión - Subdimensión & CECOSF y CESFAM (n= 87) & \multicolumn{2}{c|}{ COSAM (n = 18) } \\
\hline Primer contacto- acceso & Media (SE) & IC 95\% & Media (SE) & IC 95\% \\
\hline Continuidad atención & $2,92(0,04)$ & $2,85-3,00$ & $2,80(0,11)$ & $2,58-3,03$ \\
\hline Coordinación & $2,95(0,04)$ & $2,88-3,02$ & $3,51(0,07)$ & $3,37-3,65$ \\
\hline Coordinación - Sistema Información & $2,85(0,05)$ & $2,75-2,96$ & $3,10(0,10)$ & $2,88-3,32$ \\
\hline Integralidad - Servicios Disponibles & $3,41(0,05)$ & $3,31-3,50$ & $3,11(0,13)$ & $2,84-3,39$ \\
\hline Integralidad - Servicios Provistos & $3,48(0,03)$ & $3,44-3,53$ & $1,65(0,06)$ & $1,52-1,78$ \\
\hline Enfoque familiar & $3,17(0,05)$ & $3,06-3,28$ & $2,80(0,13)$ & $2,53-3,07$ \\
\hline Orientación comunitaria & $3,45(0,05)$ & $3,35-3,55$ & $3,64(0,08)$ & $3,48-3,80$ \\
\hline Competencia cultural & $3,36(0,05)$ & $3,26-3,45$ & $2,97(0,11)$ & $2,73-3,21$ \\
\hline Puntaje esencial * & $2,85(0,06)$ & $2,74-2,96$ & $2,51(0,10)$ & $2,30-2,71$ \\
\hline Puntaje general** & $3,13(0,03)$ & $3,08-3,19$ & $2,83(0,07)$ & $2,69-2,97$ \\
\hline
\end{tabular}

*Promedio de los puntajes de las cuatro dimensiones básicas: primer contacto, continuidad, coordinación, integralidad; **Promedio de los puntajes de las siete dimensiones. Fuente: PCAT Prestadores, Conchalí 2017-18.

obtenidos de los CESFAM y CECOSF, de los del COSAM, el puntaje correspondiente al enfoque familiar en los primeros se mantuvo alto $(3,45$; $\left.\mathrm{IC}_{95 \%} 3,35-3,55\right)$, aunque inferior al obtenido por servicios disponibles (subdominio de la integralidad) $\left(3,48 ; \mathrm{IC}_{95 \%} 3,44-3,53\right)$. En cambio, en el COSAM este aspecto obtuvo un puntaje extremadamente bajo $\left(1,65 ; \mathrm{IC}_{95 \%} 1,52-1,78\right)$; dada su especialización, estos centros no brindan todas las prestaciones consideradas en el PCAT, por lo que la evaluación de esta dimensión debe ser tomada con precaución.
Los puntajes asignados al enfoque familiar fueron consistentemente altos; el promedio del $\operatorname{COSAM}\left(3,64 ; \mathrm{IC}_{95 \%} 3,48-3,80\right)$ fue superior al de los otros centros $\left(3,45 ; \mathrm{IC}_{95 \%} 3,35-3,55\right)$, sin ser estadísticamente significativo. La competencia cultural fue evaluada con promedios inferiores a 3 puntos en todos los establecimientos.

En la Tabla 4 se enlistan los ítems del cuestionario que obtienen los puntajes más altos y más bajos en cada dominio, que permiten identificar aspectos específicos del funcionamiento del sistema comunal de salud. 
Tabla 4. Preguntas con promedios de puntaje más altos y más bajos en cada dominio

\begin{tabular}{|c|c|c|}
\hline Dominio & Puntajes más altos & Puntajes más bajos \\
\hline $\begin{array}{l}\text { Primer } \\
\text { contacto }\end{array}$ & $\begin{array}{l}\text { - Horario apertura hasta 20:00 }(3,87) \\
\text { - Lugar cercano atención nocturna }(3,65)\end{array}$ & $\begin{array}{l}\text { - Atención telefónica cuando centro está cerrado } \\
(1,91)\end{array}$ \\
\hline Continuidad & $\begin{array}{l}\text { - Entiende preguntas del paciente }(3,85) \\
\text { - Paciente se siente cómodo hablando }(3,53)\end{array}$ & $\begin{array}{l}\text { - Llamar para consultar dudas }(1,92) \\
\text { - Atención por mismo médico }(2,60)\end{array}$ \\
\hline Coordinación & $\begin{array}{l}\text { - Informa a pacientes sobre diferentes lugares } \\
\text { para derivación o interconsulta }(3,43)\end{array}$ & $\begin{array}{l}\text { - Informe telefónico o email resultados laborato- } \\
\text { rio }(2,01) \\
\text { - Conoce atenciones de especialidad que reciben } \\
\text { sus pacientes }(2,50)\end{array}$ \\
\hline $\begin{array}{l}\text { Coordinación } \\
\text { - sistema } \\
\text { información }\end{array}$ & $\begin{array}{l}\text { - Fichas clínicas disponibles cuando atiende }(3,84) \\
\text { - Lista medicamentos en uso en ficha clínica } \\
(3,61)\end{array}$ & $\begin{array}{l}\text { - Auditorías periódicas fichas clínicas }(2,59) \\
\text { - Guía manejo clínico en ficha clínica }(2,85)\end{array}$ \\
\hline $\begin{array}{l}\text { Integralidad } \\
\text { Servicios } \\
\text { disponibles }\end{array}$ & $\begin{array}{l}\text { - Consejería problemas de conducta /salud mental } \\
(3,88) \\
\text { - Consejería o tratamiento abuso sustancias o } \\
\text { drogas }(3,84)\end{array}$ & $\begin{array}{l}\text { - Examen de audición }(1,66) \\
\text { - Inmunoterapia }(1,76)\end{array}$ \\
\hline $\begin{array}{l}\text { Integralidad } \\
\text { Servicios } \\
\text { provistos }\end{array}$ & $\begin{array}{l}\text { - Temas conversados con paciente: medicamentos } \\
\text { en uso }(3,87) \text {; alimentación saludable }(3,77) \text { y } \\
\text { consejo sobre ejercicio físico }(3,69)\end{array}$ & $\begin{array}{l}\text { - Temas conversados con paciente: tenencia de } \\
\text { armas }(1,83) \text {, uso cinturón de seguridad y casco } \\
\text { bicicleta en niños }(2,19)\end{array}$ \\
\hline $\begin{array}{l}\text { Enfoque } \\
\text { familiar }\end{array}$ & $\begin{array}{l}\text { - Pregunta al paciente sobre problemas de salud } \\
\text { en familia }(3,80) \\
\text { - Discusión sobre factores de riesgo familiares } \\
(3,57)\end{array}$ & $\begin{array}{l}\text { - Evaluación signos abuso infantil }(3,34) \\
\text { - Discusión sobre condiciones de vivienda }(3,36)\end{array}$ \\
\hline $\begin{array}{l}\text { Orientación } \\
\text { comunitaria }\end{array}$ & $\begin{array}{l}\text { - Realiza visitas domiciliarias }(3,98) \\
\text { - Trabajo con juntas de vecinos/ líderes comunita- } \\
\text { rios }(3,60)\end{array}$ & $\begin{array}{l}\text { - Vínculo con organizaciones religiosas }(2,57) \\
\text { - Uso datos de riesgos salud ocupacional para } \\
\text { programas }(2,68)\end{array}$ \\
\hline $\begin{array}{l}\text { Competencia } \\
\text { intercultural }\end{array}$ & $\begin{array}{l}\text { - Considera creencias de la familia sobre trata- } \\
\text { miento /medicina tradicional }(3,43) \\
\text { - Considera solicitud de usar medicina alternativa } \\
\text { (homeopatía, acupuntura) }(3,40)\end{array}$ & $\begin{array}{l}\text { - Entrenamiento equipo salud con instructores } \\
\text { externos para abordar diversidad cultural }(1,99) \\
\text { - Capacitación por profesionales propios para } \\
\text { abordar diversidad cultural }(2,15)\end{array}$ \\
\hline
\end{tabular}

Fuente: PCAT Prestadores, Conchalí 2017-18.

\section{Discusión}

Tanto el puntaje esencial como el general, y los promedios de cada uno de los dominios, salvo el de competencia cultural, son superiores al valor 3 , considerado como "buen desempeño"16. Estos puntajes, numéricamente altos, deben analizarse considerando las particularidades de los aspectos que los componen.

El alto puntaje obtenido por la integralidad de los servicios contrasta con la percepción de los prestadores, de que la mitad de sus pacientes no va a ese centro para atender todas sus necesidades de salud, lo que podría relacionarse con una falta de acceso a las prestaciones disponibles. Esta brecha entre disponibilidad y acceso a servicios es clave $^{28}$, aunque no fue posible evaluarla, porque el instrumento no incluye iguales prestaciones en los subdominios servicios disponibles y servicios provistos.

Entre los servicios disponibles destacan las consejerías sobre abuso de sustancias y problemas de salud mental, y en servicios provistos las referidas a medicación en uso, alimentación y ejercicio físico. La prevención de otros riesgos, como el uso del cinturón de seguridad y del casco para bicicleta, o la tenencia de armas, son poco discutidos en la consulta. Esto sugiere una práctica con enfoque más individual que colectivo y social ${ }^{29}$, aunque este último constituye un elemento clave del MAIS ${ }^{1}$.

El buen desempeño del dominio enfoque familiar debe ser analizado con cautela, ya que muchas 
de sus preguntas se orientan a detectar riesgos y discutir problemas en torno a crisis familiares que afectan la salud, más que a su reconocimiento e incorporación como recurso terapéutico.

En cuanto a la orientación comunitaria, las actividades incluidas en las normativas institucionales (visitas domiciliarias, trabajo con juntas de vecinos, evaluación de programas por los equipos de salud) presentan mejores evaluaciones que las no incluidas (vínculo con organizaciones religiosas, existencia y uso de datos sobre salud ocupacional, entre otros), lo que cuestiona la real adaptación de la APS al contexto local ${ }^{7,30}$.

La valoración del dominio competencia cultural parece estar asociada a una preocupación ante el incremento de la inmigración ${ }^{31}$, especialmente de población haitiana, y en menor medida, al debate nacional sobre la situación de los pueblos originarios $^{32}$. Algunos de los ítems de este dominio son planteados en términos amplios y no necesariamente implican la incorporación de la diversidad cultural en el manejo clínico.

Los encuestados consideran que están en capacidad de atender a personas de otros contextos culturales, pero piden mayor capacitación en esa temática. Además, solicitan mayor número de intérpretes y facilitadores culturales. Destaca la necesidad de facilitar la comunicación entre prestadores y usuarios inmigrantes, y de fortalecer las competencias interculturales de los profesionales de la salud.

Por otra parte, el dominio de continuidad de atención se evalúa como "buen desempeño" en todos los establecimientos. Aquellos ítems referidos a la comunicación prestador-paciente (entiende las preguntas del paciente, paciente se siente cómodo hablando de sus problemas con usted) alcanzan puntajes más altos que aquellos sobre la continuidad intraprestador (si los usuarios son atendidos por el mismo médico). La insuficiente continuidad también se refleja en la caracterización de los prestadores de la población atendida. La continuidad es primordial en la calidad de la atención ${ }^{33}$, especialmente cuando un alto porcentaje de pacientes tiene patologías crónicas ${ }^{34}$. Para reforzarla, Chile implementó la sectorialización y los registros computarizados que identifican al paciente con su prestador ${ }^{1}$.

Vinculada a los registros computarizados, la evaluación del subdominio coordinación-sistemas de información es alta, porque sus ítems se refieren a la disponibilidad de información dentro de la ficha clínica. La transmisión de información a otros niveles de atención es evaluada en el dominio coordinación, cuyos ítems referidos a la recepción de resultados de laboratorio por vía telefónica o correo electrónico, y de información sobre atenciones especializadas, son los peor evaluados, reflejando la fragmentación de este sistema ${ }^{35}$.

Las diferencias en los puntajes obtenidos por el COSAM en varias dimensiones son explicables dada su especialización: sus servicios disponibles están limitados al ámbito de la salud mental y su funcionamiento difiere de los otros establecimientos de la red, aunque mantiene y fortalece los valores y principios claves de la APS, siendo entendido así por los demás integrantes del sistema. Las variaciones en los puntajes de los dominios orientación comunitaria, competencia cultural y continuidad de la atención ameritan investigaciones que profundicen en sus causas y permitan adaptar el instrumento a los COSAM.

Estos resultados deben ser analizados a la luz de lo descrito en las primeras validaciones del PCAT, que mostraban que la distribución de las respuestas siempre tiende hacia las más favorables (puntaje $\geq 2,5)^{8}$.

Las aplicaciones del PCAT generalmente incluyen a prestadores y usuarios ${ }^{36-42}$; siendo la percepción de los primeros consistentemente más positiva que la segunda ${ }^{42}$, especialmente en lo referido a coordinación, enfoque familiar y, en menor medida, orientación a la comunidad; la puerta de entrada es el ítem con más alto puntaje. Esto coincide con los resultados del estudio de van Stralen, en Brasil: la mayor brecha entre la percepción de los usuarios y los prestadores está en el enfoque familiar y comunitario ${ }^{40}$.

Por el importante papel que desempeñan los técnicos de salud en la APS chilena, sería interesante aplicar el PCAT-Prestadores a no profesionales, como lo hizo un estudio realizado en Quebec ${ }^{39}$. Esto requiere de su adaptación y validación con este grupo específico, que aún no se ha realizado en nuestro país.

Dada la baja e irregular tasa de respuesta de los prestadores, los resultados de esta primera aplicación del PCAT en un sistema municipal de salud chileno no pueden ser extrapolados fuera de la muestra obtenida. Esta tasa fue particularmente baja entre los médicos, quienes desempeñan un rol clave dentro del proceso de atención y 
en la interacción con el paciente, lo que implica una limitación. Los reportes de aplicación del PCAT-Prestadores no presentan resultados desagregados por profesión, salvo el de Silva y colaboradores $^{43}$, pero se ha reportado baja disposición de los médicos para registrar información sobre calidad de atención, probablemente asociada a su alta carga asistencial ${ }^{44}$.

El alto porcentaje de encuestados que pueden ser considerados personal estable (más de cinco años trabajando en APS, jornada completa, remuneración por sueldo) contrasta con la conocida movilidad laboral de los profesionales, y especialmente los médicos, en $\mathrm{APS}^{45}$; fenómeno que debería ser explorado a profundidad. Aun con estas limitaciones, esta investigación permitió obtener valiosa información acerca del funcionamiento de la APS en Conchalí, que aporta pistas para futuras intervenciones e investigaciones sobre este importante componente del sistema de salud.

Se recomienda completar este estudio con la aplicación del PCAT a prestadores no profesionales y a usuarios, a fin de comparar la información, así como contrastar estos resultados con los obtenidos en otras comunas o regiones contribuyendo a la implementación del MAIS en Chile.

Agradecimientos: A la Dirección de Salud de la Corporación Municipal de Conchalí (CORESAM) por permitir la realización de esta investigación en su red de establecimientos de salud.

\section{Referencias}

1. Ministerio de Salud. Orientaciones para la implementación del modelo de atención integral de salud familiar y comunitaria. Dirigido a equipos de salud [Internet]. Minsal. Santiago de Chile; 2013. Available from: http://web.minsal.cl/portal/url/item/e7b24eef3e5cb5d1e0400101650128e9.pdf

2. Ministerio de Salud. Modelo de Atención Integral en Salud. Santiago de Chile; 2005. (Cuadernos Modelo de Atención). Report No.: 1.

3. Organización Panamericana de la Salud. La Renovación de la Atención Primaria de Salud en las Américas Documento de Posición de la Organización Panamericana de la Salud / Organización Mundial de la Salud. Washington D.C.; 2007.

4. World Health Organization. Quality of care: A process for making strategic choices in health systems. France: World Health Organization; 2006.

5. Kruk M, Kelly E, Syed S, Tarp F, Addison T. Measuring quality of health-care services: what is know and what are the gaps. WHO Bull [Internet] 2017; 95: 389. Available from: http://dx.doi.org/10.2471/BLT.17.195099.

6. Hanefeld J, Powell-Jackson T, Balabanova D. Understanding and measuring quality of care: dealing with complexity. Bull World Heal Organ 2017; 95: 368-74.

7. Primary Health Care Performance Initiative. Methodology Note: [Internet]. Methodology Note. 2015. Available from: https://www.phcperformanceinitiative.org/

8. Shi L, Starfield B, Xu J. Validating the adult primary care assessment tool. J Fam Pract [Internet]. 2001; 50 (2): 161-75.

9. The Johns Hopkins Primary Care Policy Center. Primary Care Assessment Tools [Internet]. [citado el 24 de mayo de 2017]. Disponible en: http://www.jhsph. edu/research/centers-and-institutes/johns-hopkins-primary-care-policy-center/pca_tools.html.

10. Pasarín MI, Berra S, González A, Segura A, Tebé C, García-Altés A, et al. Evaluation of primary care: The "Primary Care Assessment Tools-Facility version"for the Spanish health system. Gac Sanit [Internet] 2013; 27 (1): 12-8.

11. Bresick G, Sayed AR, Le Grange C, Bhagwan S, Manga $\mathrm{N}$. Adaptation and cross-cultural validation of the United States Primary Care Assessment Tool (expanded version) for use in South Africa. African J Prim Heal Care Fam Med 2015; 7 (1): 1-11.

12. Mei J, Liang Y, Shi L, Zhao J, Wang Y, Kuang L. The Development and Validation of a Rapid Assessment Tool of Primary Care in China. BioMed Research International 2016. Disponible en http://dx.doi.org/10.1155/ 2016/6019603.

13. Harzheim E, Starfield B, Rajmil L, Álvarez-Dardet C, Stein AT. Consistência interna e confiabilidade da versão em português do Instrumento de Avaliação da Atenção Primária (PCATool-Brasil) para serviços de saúde infantil. Cad Saude Publica [Internet] 2006; 22 (8): 1649-59.

14. Pizzanelli M, Ponzo J, Buglioli M, Toledo A, Casinelli M. Validación de Primary Care Assessment Tool ( PCAT ) en Uruguay. Rev Med Urug 2011; 27 (3): 187-9.

15. Berra S, Hauser L, Audisio Y, Mántaras J, Nicora V, Oliveira MMC de, et al. Validez y fiabilidad de la versión argentina del PCAT-AE para evaluar la atención primaria de salud. Rev Panam Salud Pública [Internet] 2013; 33 (1): 30-9.

16. Escuela de Salud Publica - Facultad de Medicina - Universidad de Chile. Guía de recomendación y uso del 
Instrumento Primary Care Assessment Tools (PCAT) versiones "usuario adulto" y "prestadores" para la Atención Primaria de Salud de Chile. Santiago de Chile; 2016.

17. Departamento de Estadísticas e Información de Salud; Ministerio de Salud; Gobierno de Chile. Atenciones de la Red Asistencial Pública [Internet]. [citado el 3 de febrero de 2018]. Disponible en: http://www.deis.cl/ estadisticas-redpublica/

18. Ministerio de Salud. Orientaciones Técnicas y Metodológicas Compromisos Gestión en el marco de las Redes Integradas de Servicios Salud. Santiago de Chile; 2016.

19. Ministerio de Salud; Subsecretaría de Salud Pública. Ley 20.645: Crea asignación asociada al mejoramiento de la calidad de trato al usuario, para los funcionarios regidos por el estatuto de Atención Primaria de Salud Municipal [Internet]. Chile: Biblioteca del Congreso Nacional; 2012. Disponible en: www.leychile.cl.

20. Superintendencia de Salud -Gobierno de Chile. Registros de entidades reguladas por la Superintendencia de Salud. [Internet]. [citado el 3 de febrero de 2018]. Disponible en: http://www.supersalud.gob.cl/acreditacion/673/w3-channel.html.

21. Muñoz D, Pontoja T, Undurraga J. La calidad del cuidado de salud en Atención Primaria: Desarollo y validación de un instrumento para la medición técnica. Rev Chil Med Fam 2004; 5 (3): 118-27.

22. Leisewitz T, Nogueira L, Peñaloza B, Bastías G, Villarroel L. Evaluación de los servicios de salud primaria en Chile: Perspectiva del paciente-Proyecto FONIS SA07120032. Santiago de Chile; 2011.

23. Servicio de Salud Metropolitano Norte. Diagnóstico de Situación de Salud Área de Influencia. 2013.

24. Sistema Nacional de Información Municipal; Subsecretaría de Desarrollo Regional y Administrativo. Ficha Comunal Conchalí [Internet]. [citado el 1 de julio de 2017]. Disponible en: http://datos.sinim.gov.cl/ficha_comunal.php\#tab-antecedentes-municipales.

25. Ministerio de Desarrollo Social- Gobierno de Chile. Encuesta Casen-Observatorio Social [Internet]. 2017 [citado el 25 de octubre de 2018]. Disponible en: http://observatorio.ministeriodesarrollosocial.gob.cl/ casen-multidimensional/casen/casen_2017.php.

26. Servicio de Salud Metropolitano Norte. Conchalí en Cifras [Internet]. 2013. Disponible en: http://www.ssmn. cl/descargas/estadistica/estadistica_Conchali.pdf

27. Servicio de Salud Metropolitano Norte. Red de Establecimientos [Internet]. [citado el 14 de agosto de 2017]. Disponible en: http://www.ssmn.cl/red_establecimientos.php.

28. World Health Organization. Tracking Universal Health
Coverage: 2017 Global Monitoring [Internet]. 2017. 1-88 p. Disponible en: http://pubdocs.worldbank.org/ en/193371513169798347/2017-global-monitoring-report.pdf.

29. Maeseneer M, Willems S, De Sutter M, Van de Geucchte I, Billings M. Primary health care as a strategy for achieving equitable care: a literature review commissioned by the Health Systems Knowledge Network. WHO Commission on the Social Determinants of Health. 2007.

30. Kruk ME, Nigenda G, Knaul FM. Redesigning primary care to tackle the global epidemic of noncommunicable disease. Am J Public Health 2015; 105 (3): 431-7.

31. Bernales M, Cabieses B, McIntyre AM, Chepo M. Desafíos en la atención sanitaria de migrantes internacionales en Chile. Rev Peru Med Exp Salud Publica 2017; 34 (2): 167-75.

32. Cuyul Soto A. La política de salud chilena y el pueblo Mapuche. Entre el multiculturalismo y la autonomía mapuche en salud. Salud Probl 2013; 14: 21-33.

33. Dois A, Bravo P, Soto G. Atributos y características de los principios orientadores del Modelo de Atención Integral de Salud Familiar y Comunitaria desde la perspectiva de expertos en APS. Rev Med Chile [Internet]. 2017; 145 (7): 879-87.

34. Ministerio de Salud. Documento presentación primeros resultados Tercera Encuesta Nacional de Salud (ENS) 2016-2017 [Internet]. Santiago de Chile ; 2018 [citado el 18 de octubre de 2018]. Disponible en: http://epi. minsal.cl/wp-content/uploads/2017/12/2017.21.07_pdf. primeros.resultados.pdf.

35. Dois A, Contreras A, Bravo P, Mora I, Soto G, Solís C. Principios orientadores del Modelo Integral de Salud Familiar y Comunitario desde la perspectiva de los usuarios. Rev Med Chile [Internet]. 2016; 144: 585-92.

36. Muldoon L, Dahrouge S, Hogg W, Geneau R, Russell G, Shortt M. Community orientation in primary care practices: Results from the Comparison of Models of Primary Health Care in Ontario Study. Can Fam Physician [Internet]. 2010; 56 (7): 676-83.

37. Rowan MS, Lawson B, MacLean C, Burge F. Upholding the principles of primary care in preceptors' practices. Fam Med 2002; 34 (10): 744-9.

38. Russell G, Dahrouge S, Tuna M, Hogg W, Geneau R, Gebremichael G. Getting it all done. Organizational factors linked with comprehensive primary care. Fam Pract 2010; 27 (5): 535-41.

39. Haggerty JL, Pineault R, Beaulieu M-D, Brunelle Y, Gauthier J, Goulet F, et al. Practice Features Associated With Patient reported Accessibility, Continuity, and Coordination of Primary Health Care. Ann Fam Med 2008; 6 (2): 116-24. 
40. van Stralen CJ, Belisário SA, van Stralen TBDS, Lima AMD De, Massote AW, Oliveira CDL. Perceptions of primary health care among users and health professionals: a comparison of units with and without family health care in Central-West Brazil. Cad saude publica/ Minist da Saude, Fund Oswaldo Cruz, Esc Nac Saude Publica 2008; 24 Suppl 1: S148-58.

41. Rodríguez-Villamizar LA, Acosta-Ramírez N, Ruiz-Rodríguez M. Evaluación del desempeño de servicios de Atención Primaria en Salud: experiencia en municipios rurales en Santander, Colombia. Rev Salud Publica 2013; 15 (2): 167-79.

42. Vega-Romero R, Martínez Collantes J, Acosta Ramírez N. Evaluación rápida del desempeño de la red pública de servicios de salud de Suba en el logro de los atributos de la Atención Primaria de Salud. Rev Gerenc Polít Salud
[Internet] 2009; 8 (16): 165-90.

43. Silva SA da, Nogueira DA, Paraizo CM da S, Fracolli LA, Silva SA da, Nogueira DA, et al. Assessment of primary health care: health professionals' perspective. Rev da Esc Enferm da USP [Internet]. 2014 Aug [citado el 23 de enero de 2019]; 48 (spe): 122-8.

44. Alameddine M, Saleh S, Natafgi N. Assessing health-care providers' readiness for reporting quality and patient safety indicators at primary health-care centres in Lebanon: a national cross-sectional survey. Hum Resour Health [Internet]. 2015 [citado el 4 de enero de 2019];13: 37.

45. Bass C, Guerrero-Montofre I, Villacís-Coronel F, Ulloa Gutiérrez O, Kremser Ruiz I. Política Integral para el Recurso Médico de la Atención Primaria de Chile. Cuad Médico Soc 2013; 53 (2): 105-16. 